\title{
MRST: A TOOL FOR THE STORAGE OF MICRORNA COMPUTATIONAL RESOURCES
}

\author{
MING CHEN 2,3, ZHONGYANG TAN ${ }^{1, *}$, GUANGMING ZENG $2,3,{ }^{*}$, MINGFU LI ${ }^{4}$ \\ ${ }^{1}$ College of Biology, State Key Laboratory for Chemo/Biosensing and Chemometrics, Hunan University, Changsha \\ 410082, China \\ ${ }^{2}$ College of Environmental Science and Engineering, Hunan University, Changsha 410082, China \\ ${ }^{3}$ Key Laboratory of Environmental Biology and Pollution Control (Hunan University), Ministry of Education, Changsha \\ 410082, China \\ ${ }^{4}$ Chinese Academy of Inspection and Quarantine Beijing, 100029, China \\ *Corresponding Author: Email- zhongyang@hnu.cn (Z. Tan) and zgming@hnu.cn (G. Zeng)
}

Received: April 05, 2011; Accepted: April 20, 2011

\begin{abstract}
More and more computational resources for microRNAs (miRNAs) have been developed in recent years. That may lead to selective difficulty, mistakes of usage and confusion due to their confined scope of usage. Here, we summarize and classify the knowledge that has been accumulated in the fields and present a novel tool, MRST (miRNA resource store tool), for the storage of miRNA resources.
\end{abstract}

Key words - microRNA; target gene; computational algorithm.

\section{Introduction}

MicroRNAs (miRNAs) are short non-coding RNAs with the length of $22 \mathrm{nt}$ approximately, and sever a functional role that either results in the degradation of mRNAs or suppresses their translation by binding to the 3'-untranslated region (3'-UTR) [1,2]. To date, there are large number of miRNA sequences have been found $[3,4]$. First, a long primary miRNAs (primiRNAs) are processed into 60-70 nt hairpin-like precursor transcripts (pre-miRNAs) by Drosha, and then are cleaved into mature miRNAs by another RNase III, Dicer [5]. It has been demonstrated that miRNAs play important roles in various biological processes, such as cell proliferation, differentiation, development, diseases, transcriptional gene regulatory network, neuronal synapses formation, and cell death $[5,6]$. Some computational tools for the identification of miRNA gene have been developed, and so far, the two most sensitive tools are MiRscan and miRseeker [7-9]. It is believed that functional understanding of miRNAs will depend heavily on identification of their corresponding target mRNAs [10]. MiRNA target predictions in animals are thought to be more difficult than in plants because miRNAs of animals are short and miRNA-mRNA duplexes are not entirely complementary to one another [11]. Computational algorithms have been developed based on extracted principles such as sequence complementarity, thermodynamic stability calculations and evolutionary conservation among species $[5,10]$. Moreover, it is also suggested stronger binding at the 3 ' region compensate for imperfect base pairing within the seed segment, and a method has considered the contribution of 3 ' region in target identification [12].
Due to accumulating knowledge of miRNAs and their target genes, some useful databases have been constructed, of which miRBase serves as a registry of the information of miRNA sequences [3].

Large number of computational tools and databases are developed, leading to selective difficulty for the usage due to the lack of classification and conclusion. Therefore, users must determine appropriate methods or tools according to what they wish to gain from a given analysis. These situations suggest that the performance of currently available algorithms or databases may need to be assembled and sorted. All these encourage us to seek solutions to improve the use effect of miRNA resources.

We present here, a tool for the collection with existed computational tools and databases of miRNAs. In our approach, data consistency and data redundancy are considered. We believe that our work will contribute significantly to facilitate the usage of these tools and databases. Most important, it facilitates the research on the roles of miRNAs.

\section{Materials and methods}

There are numerously useful resources and available software tools for analysis of miRNAs, as summarized in Table 1. We conclude the efforts made by different software tools to predict miRNA genes or target genes, their principles of miRNA target recognition, and their supported organisms for computational prediction. This comprehensive collection with tools and databases of miRNAs is expected to provide an overall view of the knowledge that has been 
accumulated in the field and be helpful to research of miRNAs.

\section{Data collection with databases of miRNAs and their targets}

All data and information were obtained from databases and literature. After finishing data collection, the second step involves mining information from various datasets. Because different databases and computational tools own various limits and scopes of usage. To resolve this problem, a variety of databases and related literature are assessed, and the main features of these resources are investigated. Table 1 presents the data collected which are preprocessed according to the mainly advanced features. The websites of these tools are given in Table 2 and this software. Previous works introduced and reviewed some of these databases, but the knowledge of most databases is not mentioned and sorted out $[10,11]$. The present work will facilitate the comparisons between different databases.

Data collection with computational algorithms for identification and prediction of miRNA genes and miRNA targets

Supported organisms of various software tools may be non-identical. These tools are investigated, and are classified as 'any', 'animal', 'vertebrates', 'flies', 'nematodes', 'viruses', and 'plants' according to their supported organisms $[10,11]$. Main features, websites and references of these tools are shown in Tables 1 and 2.

\section{Construction of software}

MRST is composed of three modules: databases of miRNAs and their targets, computational algorithms for miRNA gene identification, and computational algorithms for miRNA target prediction. The proposed procedure interface provides a variety of available websites of reported databases and computational algorithms for both browsing functions and search functions. The detailed introduction is provided in this tool.

\section{Implementation}

MRST has been developed in standard Microsoft visual basic language. The program collects most of available databases and computational tools of miRNAs. The program provides four options for users: introduction of computational resources; available online resources for databases of miRNAs; available online resources for computational algorithms of miRNA gene identification; and available online resources for computational algorithms of miRNA target prediction. A sand-alone application with a user's guide is available for free access at http://hudacm11.mysinamail.com/bioinformatics.html. Moreover, this software is available from authors Zhongyang Tan and Guangming Zeng on request (zhongyang@hnu.cn; zgming@hnu.cn).

Results and discussion
MiRNAs have been proved to be involved in a variety of biological processes. It is important to collect widely available algorithms for research of miRNAs. This work will help a lot to broaden our understanding with computational resources of miRNAs, and presents a novel tool for the storage of miRNA resources including databases of miRNAs and their targets, computational algorithms for miRNA gene identification, computational algorithms for miRNA target prediction. We believe that the proposed tool can provide sufficient and effective information for the investigation about availably computational resources of miRNAs. Alternatively, the proposed tool allows users to search databases of miRNAs or find the most suitable software tools for their analysis that is more convenient.

\section{Acknowledgement}

The authors sincerely thank Editor and anonymous reviewer for suggestions on improving the paper. The study was financially supported by Production, Education and Research guiding project, Guangdong Province (2010B090400439), Great program for GMO, Ministry of Agriculture of the people Republic of China (2009ZX08015-003A), the National Natural Science Foundation of China (No. 50608029, No.50978088, No. 50808073, No.51039001), Hunan Provincial Innovation Foundation for Postgraduate, the National Basic Research Program (973 Program) (No. 2005CB724203), Program for Changjiang Scholars and Innovative Research Team in University (IRT0719), the Hunan Provincial Natural Science Foundation of China (10JJ7005), the Hunan Key Scientific Research Project (2009FJ1010), and Hunan Provincial Innovation Foundation For Postgraduate (CX2010B157).

\section{References}

[1] Megraw M., Sethupathy P., Corda B. and Hatzigeorgiou A.G. (2007) Nucleic Acids Res 35, D149-D155.

[2] John B., Enright A.J., Aravin A., Tuschl T., Sander C. and Marks D.S. (2004) PLoS Biol 2, e363.

[3] Griffiths-Jones S., Grocock R.J., van Dongen S., Bateman A. and Enright A.J. (2006). Nucleic Acids Res 34, D140-D144.

[4] Chen M., Tan Z., Zeng G. and Peng J. (2010) Mol Biol Evol 27, 2227-2232.

[5] Bartel D.P. (2004) Cell, 116, 281-297.

[6] Hsu P.W. et al. (2006) Nucleic Acids Res 34, D135-D139.

[7] Lim L.P., Lau N.C., Weinstein E.G., Abdelhakim A., Yekta S., Rhoades M.W., Burge C.B. and Bartel D.P. (2003) Genes Dev 17, 991-1008.

[8] Lim L.P., Glasner M.E., Yekta S., Burge C.B. and Bartel D.P. (2003) Science 299, 1540. 
[9] Lai E.C., Tomancak P., Williams R.W. and Rubin G.M. (2003) Genome Biol 4, R42.

[10] Watanabe Y., Tomita M. and Kanai A. (2007) Methods Enzymol 427, 65-86.

[11] Rajewsky N. (2006) Nat Genet 38 Suppl, S8-13.

[12] Yousef M., Jung S., Kossenkov A.V., Showe L.C. and Showe M.K. (2007) Bioinformatics 23, 2987-2992.

[13] Wang X. (2008) RNA 14, 1012-1017.

[14] Xiao F., Zuo Z., Cai G., Kang S., Gao X. and Li T. (2009) Nucleic Acids Res 37, D105D110.

[15] Chiromatzo A.O. et al. (2007) Genet Mol Res 6, 859-865.

[16] Lagana A. et al. (2009) Database (Oxford) 2009, bap008.

[17] Bao L., Zhou M., Wu L., Lu L., Goldowitz D., Williams R.W. and Cui Y. (2007) Nucleic Acids Res 35, D51-D54.

[18] Li S.C., Shiau C.K. and Lin W.C. (2008) Nucleic Acids Res 36, D184-D189.

[19] Thadani R. and Tammi M.T. (2006) BMC Bioinformatics 7 Suppl 5, S20.

[20] Hsu P.W., Lin L.Z., Hsu S.D., Hsu J.B. and Huang H.D. (2007) Nucleic Acids Res 35, D381-D385.

[21] Bandyopadhyay S. and Mitra R. (2009). Bioinformatics 25, 2625-2631 
Table 1 - Available online resources for microRNAs:

\begin{tabular}{|c|c|c|}
\hline Database/tool & Description of advantages & Reference (s) \\
\hline \multicolumn{3}{|l|}{ Databases } \\
\hline miRDB & MiRNA target prediction and functional annotation & [13] \\
\hline miRecords & An integrated resource for microRNA-target interactions & [14] \\
\hline miRGen & Relationship between animal microRNA genomic organization and function & [1] \\
\hline miRNApath & Relationships between the target genes, miRNAs and metabolic pathways & [15] \\
\hline miRò & $\begin{array}{l}\text { A web-based knowledge base that provides users with miRNA-phenotype } \\
\text { associations in humans }\end{array}$ & [16] \\
\hline PolymiRTS & Linking polymorphisms in microRNA target sites with complex traits & [17] \\
\hline Vir-Mir db & A database containing predicted viral miRNA candidate hairpins & [18] \\
\hline \multicolumn{3}{|c|}{$\begin{array}{l}\text { Tool for miRNA gene } \\
\text { identification }\end{array}$} \\
\hline MiRscan & $\begin{array}{l}\text { Relies on the observation that the known miRNAs derive from } \\
\text { phylogenetically conserved stem loop precursor RNAs with characteristic } \\
\text { features }\end{array}$ & {$[7-8]$} \\
\hline \multicolumn{3}{|c|}{$\begin{array}{l}\text { Tools for miRNA } \\
\text { target prediction }\end{array}$} \\
\hline MicroTar & $\begin{array}{l}\text { Uses predicted free energies of unbound mRNA and putative mRNA-miRNA } \\
\text { heterodimers, implicitly addressing the accessibility of the mRNA } 3^{\prime} \\
\text { untranslated region }\end{array}$ & [19] \\
\hline NBmiRTar & $\begin{array}{l}\text { Uses machine learning by a naïve Bayes classifierand both the 'seed' and } \\
\text { 'out-seed' segments of the miRNA:mRNA duplex for target identification }\end{array}$ & [12] \\
\hline ViTa & $\begin{array}{l}\text { Curate the known virus miRNA genes and the known/putative target sites of } \\
\text { human, mice, rat and chicken miRNAs }\end{array}$ & [20] \\
\hline TargetMiner & $\begin{array}{l}\text { MicroRNA target prediction with systematic identification of tissue-specific } \\
\text { negative examples }\end{array}$ & [21] \\
\hline
\end{tabular}

Note: For other miRNA databases (Argonaute, ASRP, miRBase, miRNAMap and Tarbase) and other miRNA gene and target prediction tools (Microlnspector, miTarget, RNAhybrid, GUUGle, MovingTargets, miRanda, FastCompare, TargetBoost, PicTar, rna22, miRU, TargetScanS and DIANA-microT), see [9,10]. The websites of these databases listed in this table are given in Table 2.

Table 2 - Websites of available online resources for microRNAs:

\begin{tabular}{|l|l|}
\hline Database/tool & Website \\
\hline Databases & \\
\hline miRDB & http://mirdb.org/miRDB/ \\
\hline miRecords & http://mirecords.biolead.org/ \\
\hline miRGen & http://www.diana.pcbi.upenn.edu/miRGen.html \\
\hline miRNApath & http:///gmb.fmrp.usp.br/mirnapath/ \\
\hline miRò & http://ferrolab.dmi.unict.it/miro/ \\
\hline PolymiRTS & http://compbio.utmem.edu/miRSNP/ \\
\hline Vir-Mir db & http://alk.ibms.sinica.edu.tw/cgi-bin/miRNA/miRNA.cgi \\
\hline Tool for miRNA gene identification & \\
\hline MiRscan & http://genes.mit.edu/mirscan/ \\
\hline Tools for miRNA target prediction & \\
\hline MicroTar & http://tiger.dbs.nus.edu.sg/microtar/ \\
\hline NBmiRTar & http://wotan.wistar.upenn.edu/NBmiRTar/ \\
\hline ViTa & http://vita.mbc.nctu.edu.tw/\# \\
\hline TargetMiner & http://www.isical.ac.in/ bioinfo_miu/ \\
\hline
\end{tabular}

Note: For other miRNA databases (Argonaute, ASRP, miRBase, miRNAMap and Tarbase) and other miRNA gene and target prediction tools (Microlnspector, miTarget, RNAhybrid, GUUGle, MovingTargets, miRanda, FastCompare, TargetBoost, PicTar, rna22, miRU, TargetScanS and DIANA-microT), see [9,10]. 